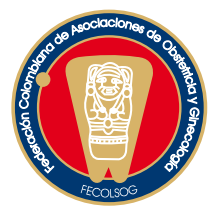

INVESTIGACIÓN ORIGINAL

\title{
ESTUDIO PILOTO DE PREVALENCIA DE INFECCIÓN POR CHLAMYDIA TRACHOMATIS DETECTADA POR PCR EN MUJERES CON PARTO PREMATURO EN EL INSTITUTO MATERNO INFANTIL DE BOGOTÁ
}

\section{A pillot study regarding the prevalence of infection caused by Chlamydia trachomatis as detected by PCR in women undergoing premature labor in the Insti- tuito Materno Infantill in Bogotá, Colombia}

Ariel Iván Ruiz, M.D., M.Sc*, Ruth Sánchez, M.Sc**, Olga Ostos, M.Sc**, Edith Angel, M.D.***, Hernando Bonilla, M.D.****, Carolina Cifuentes, M.D.*****, Constanza Correa, M.D.*****

Recibido: septiembre 1/05 - Revisado: septiembre 24/05 - Aceptado: septiembre 26/05

\section{RESUMEN}

Objetivo: determinar la prevalencia de infección por C. trachomatis en mujeres con parto prematuro.

Metodología: estudio de corte transversal en el que se analizaron 50 muestras de orina de mujeres que asistieron al Instituto Materno Infantil de Bogotá entre febrero y junio de 2004, quienes presentaron parto prematuro entre las 25 y las 36 semanas de gestación. Para su detección se utilizó la técnica de reacción en cadena de la polimerasa (PCR COBAS AMPLICOR CT, Roche diagnostic Systems).

Resultados: se encontraron dos muestras positivas para C. trachomatis, lo que corresponde a una prevalencia del 4\% (IC95\% 0,5 - 13,7) en mujeres con parto prematuro y del 4,5\% (IC95\% 0,6 - 15,5) en pacientes con RPM.

Conclusión: la $C$. trachomatis debe investigarse en pacientes con trabajo de parto prematuro o con RPM pretérmino, aún en ausencia del antecedente de flujo genital patológico y particularmente en quienes no se encuentra la causa del trabajo de parto prematuro.

Palabras clave: Chlamydia trachomatis, parto prematuro, ruptura prematura de membranas (RPM), membranas fetales, reacción en cadena de la polimerasa (PCR), prevalencia, Colombia.

\section{SUMMARY}

Objective: to determine the prevalence of Chlamydia trachomatis in women with preterm delivery.

\footnotetext{
* Ginecoobstetra, Especialista en biología de la reproducción, MSc en educación con énfasis en docencia universitaria, MSc (c) en epidemiología clínica. Profesor asociado Departamento de Obstetricia y Ginecología e Instituto de Investigaciones Clínicas. Universidad Nacional de Colombia.

Instituto Materno Infantil Cra. 10 \# 1-66 sur. Bogotá, Colombia Correo electrónico: arielruiz@supercabletv.net.co

** Bacterióloga, MSc scientae especialista en genética humana, MSc en educación con énfasis en docencia universitaria, docente investigador Universidad Colegio Mayor de Cundinamarca.
** Bacterióloga, MSc en scientae especialista en genética humana. Can- didata a doctorado en bioquímica. Docente investigador Universidad Colegio Mayor de Cundinamarca.
*** Ginecoobstetra. Fellow en infectología ginecológica y perinatal. Profe- sor asociado Departamento de Obstetricia y Ginecología Universidad Nacional de Colombia.
**** Médico especialista en epidemiología. Residente III de obstetricia y ginecología. Universidad Nacional de Colombia.
****** Residente de III año de Ginecoobstetricia. Universidad Nacional de Colombia.


Methods: cross-sectional study carried out from February to June 2004, fifty urine samples of women who assisted to the Instituto Materno Infantil of Bogotá, between the 25th and 36th weeks of gestation, were analyzed using polymerase chain reaction (PCR).

Results: two positive samples for $C$. trachomatis were found, representing a prevalence of $4 \%$ in premature delivery and $4,5 \%$ in preterm premature rupture of membranes.

Conclusion: $C$. trachomatis should be investigated in patients with premature delivery or preterm premature rupture of membranes, particularly in high-risk patients without a clinically evident cause.

Key words: Chlamydia trachomatis, premature birth, fetal membranes, premature rupture, polymerase chain reaction (PCR), prevalence, Colombia.

\section{INTRODUCCIÓN}

La Chlamydia trachomatis, es una bacteria intracelular estricta, ya que no posee las enzimas básicas para sintetizar su propio ATP. ${ }^{1}$ La bacteria parasita fundamentalmente las células epiteliales y es uno de los agentes bacterianos que causa infecciones de transmisión sexual, abortos a repetición, ruptura prematura de membranas (RPM), parto prematuro y, en el recién nacido, conjuntivitis de inclusión y neumonía. Aproximadamente el 75\% de las mujeres infectadas y el 50\% de los hombres infectados son asintomáticos. ${ }^{2}$ Las clamidias son un grupo de bacterias genéticamente diverso, que presentan un ciclo de desarrollo intracelular bifásico en el que alternan dos formas funcionales, metabólicamente diferentes: el cuerpo elemental, con capacidad infectante y el cuerpo reticulado. La C. trachomatis presenta tres biovariedades y veinte serotipos, de los cuales D, E, F, G H, I, J y $\mathrm{K}$ son los principales implicados en las patologías cervicovaginales.

El parto prematuro es un problema de salud pública. Ocurre en el 5 a 10\% de los nacimientos y es la principal causa de morbilidad y mortalidad perinatal en todo el mundo. ${ }^{3}$ En un estudio prospectivo de detección de eventos adversos en atención obstétrica y del puerperio que involucró 2.530 pacientes en el Instituto Materno Infantil, un hospital materno perinatal de referencia, se encontró una prevalencia de amenaza de parto prematuro del 37\% (IC95\% 35 - 39). ${ }^{4}$ Aunque el papel etiopatogénico de la C. trachomatis en efectos perinatales adversos como parto prematuro, RPM, bajo peso al nacer, infección intrauterina, muerte fetal intrauterina y endometritis puerperal de inicio tardío, ha sido controversial, su tratamiento oportuno reduce significativamente tales complicaciones. ${ }^{5-7}$ Un posible mecanismo por el cual se produce RPM, en infecciones causadas por C. trachomatis es la producción de prostaglandinas, que estimulan la contractibilidad uterina, así mismo colagenasas y proteasas que llevan al debilitamiento de las membranas.

La relativa dificultad para establecer el diagnóstico de la infección por Chlamydia trachomatis, se debe a que un 70 a $90 \%$ de las mujeres que presentan infección endocervical por este microorganismo son asintomáticas; además, la bacteria no crece en medios de cultivo convencionales. Los métodos usados habitualmente para la identificación de este agente son el cultivo celular, ELISA, inmunofluorescencia directa, microinmunofluorescencia indirecta y técnicas moleculares como la reacción en cadena de la ligasa (LCR) y la reacción en cadena de la polimerasa (PCR), siendo estas últimas las técnicas más específicas (90\%) y sensibles (98\%) para su detección. ${ }^{8,9}$ Una revisión sistemática reciente concluyó que los resultados de las pruebas de amplificación de ácidos nucleicos para $C$. trachomatis en muestras de orina, son casi idénticos a aquellos obtenidos en muestras obtenidas directamente del cuello uterino o de la uretra. ${ }^{10}$

El objetivo de este estudio piloto fue determinar la prevalencia de infección por $C$. trachomatis en mujeres con parto prematuro que asistieron al Instituto Materno Infantil de Bogotá, utilizando PCR para su detección, en muestras de orina. 
MATERIALES Y MÉTODOS

\section{Población y selección de la muestra}

Se realizó un estudio de corte transversal en el que participaron 50 pacientes consecutivas quienes presentaron parto entre las 25 y las 36 semanas de gestación, en el Instituto Materno Infantil (IMI) de Bogotá, entre el 1 de febrero y el 30 de junio de 2004. El IMI es una institución maternoperinatal de referencia, que atiende casos de alto riesgo obstétrico.

Los criterios de inclusión fueron: pacientes con parto espontáneo, con o sin ruptura de membranas, ocurrido entre las 25 y las 36 semanas de gestación, calculadas por fecha de última menstruación confiable o, en su defecto, por ultrasonido del primer trimestre, parto o cesárea atendidos en el Instituto Materno Infantil, y firma del consentimiento informado. Así mismo, los criterios de exclusión fueron: embarazo múltiple, malformaciones fetales mayores y óbito fetal.

\section{Detección de Chlamydia trachomatis}

La detección de $C$. trachomatis se llevó a cabo en muestras de orina, de la primera micción del primer o segundo día de puerperio. Se recolectaron 10 a 50 $\mathrm{ml}$ de orina en recipientes de polipropileno estériles, sin previo aseo vaginal y por micción espontánea para recuperar mayor cantidad de células. Las muestras fueron conservadas a $-29^{\circ} \mathrm{C}$, luego separadas en alícuotas por duplicado en tubos Ependorff $(1 \mathrm{ml})$ y refrigeradas para su posterior procesamiento en la Secretaría de Salud de Bogotá.

Las muestras se analizaron por la técnica de PCR en el equipo de COBAS (Comprehensive BioAnalytical System) AMPLICOR CT (Roche), que usa los cebadores biotinilados CP24 y CP27, para amplificar una secuencia de aproximadamente 207 pares de bases dentro del plásmido críptico, compartido por todas las serovariedades de la Chlamydia trachomatis. Una vez realizada la extracción, se realizó la amplificación del ADN con la mezcla maestra (cebadores, dNTPs, Taq Polimerasa, Cloruro de Magnesio y buffer).
Después de la PCR se realizó la reacción de hibridación, desnaturalizando los amplicones y colocándolos en pocillos de detección, que contienen las partículas magnéticas revestidas con una sonda oligonucleótida específica para el plásmido críptico de C. trachomatis. Los amplicones que están marcados con biotina, hacen hibridación con las sondas conjugadas con las partículas magnéticas.

La prueba de COBAS AMPLICOR CT emplea controles internos en paralelo, tanto para resultados positivos, como negativos.

\section{Análisis de datos}

Las variables que se midieron fueron: prevalencia de periodo, edad en años cumplidos, edad gestacional en semanas, antecedente de infecciones de transmisión sexual, uso de métodos anticonceptivos, ruptura prematura de membrana, corioamnionitis, infección puerperal y muerte neonatal. La información se recolectó desde el momento de la firma del consentimiento informado hasta la consulta de control puerperal. Las fuentes de información fueron: entrevista directa, examen clínico e historia clínica.

El análisis de datos se realizó con el programa STATA 6,0 (TX, USA) utilizando estadística descriptiva, Shapiro-Wilks para distribución de los datos e intervalos de confianza (binomial exacto).

\section{Aspectos éticos}

El protocolo de investigación fue aprobado por los comités de ética e investigaciones del Departamento de Obstetricia y Ginecología y del IMI.

\section{RESUILTADOS}

Se encontraron 2 muestras positivas para C. trachomatis, lo que representa una prevalencia del 4\% (IC95\% 0,5 - 13,7) en este grupo de pacientes. La mediana de la edad de las pacientes fue 22 años (percentil 25: 20 años, percentil 75: 27 años, rango de 15 a 34 años), las edades de las dos pacientes con muestras positivas fueron $21 \mathrm{y}$ 
33 años. El promedio de edad gestacional fue de 31,5 semanas (DE: 2,7 semanas).

En nuestro estudio 44 pacientes presentaron RPM pretérmino (88\%), de las cuales 15 presentaron RPM prolongada $(34,1 \%)$. Las dos pacientes positivas para Chlamydia tenían RPM pretérmino, lo que representa una prevalencia de 4,5\% (IC95\% 0,6 - 15,5) de infección por Chlamydia en este subgrupo.

Se estableció el diagnóstico de coriomanionitis en 11 pacientes (22\%); en ninguno de estos casos se detectó $C$. trachomatis. Tres pacientes presentaron infección de vías urinarias (6\%), estas pacientes tuvieron pruebas negativas para Chlamydia. Ninguna paciente tenía antecedente de infecciones de transmisión sexual y todas las serologías VDRL fueron negativas. Dos pacientes presentaron endometritis puerperal; ambas tenían prueba negativa para C. trachomatis. Ocurrió una muerte neonatal temprana (2\%), cuya madre fue negativa para $C$. trachomatis.

Encontramos una baja prevalencia de uso de métodos anticonceptivos en la población estudiada; 40 de 48 pacientes con prueba negativa (82\%) y una de las dos pacientes con prueba positiva no utilizaban métodos anticonceptivos. Ninguna de las dos pacientes con prueba positiva para C. trachomatis refirió flujo vaginal patológico.

\section{DISCUISIÓN}

La infección por C. trachomatis tiene una incidencia mundial anual estimada en 50 millones de casos, ${ }^{11}$ siendo esta la causa mas frecuente de infección del aparato genital femenino. ${ }^{12,13} \mathrm{Sin}$ embargo, la infección no se diagnostica en todas las pacientes, debido a que la mayoría de las infecciones son subclínicas.

En nuestro estudio la prevalencia de $C$. trachomatis fue del 4\% (IC95\% 0,5 - 13,7); un estimativo mas preciso requiere un tamaño de muestra mayor. Otra limitación del estudio proviene del hecho que no se trata de una muestra probabilística; sin embargo, nuestros datos van a permitir el cálculo apropiado de un tamaño de muestra. Por otro lado, la prevalencia señalada puede corresponder a una subestimación, ya que algunas pacientes infectadas son positivas solamente en muestras obtenidas de endocérvix, mientras que en otras solo se detecta la bacteria en muestras de orina. ${ }^{14}$ No hubo exclusiones por razones diferentes a los criterios de selección. En este estudio se utilizaron muestras de orina dado que se trataba de mujeres en puerperio inmediato. $\mathrm{Al}$ parecer una contaminación de las muestras con sangre, no inhibe significativamente el PCR cuando se compara con una muestra sin contaminación, ${ }^{15}$ por otro lado la muestra de orina se promueve por ser menos invasiva y porque podría ser utilizada para autodiagnóstico. La prevalencia informada de infección por Chlamydia depende de la población estudiada y de las características operativas del método diagnóstico. Miller y colaboradores en un estudio prospectivo que involucró 20.000 personas adolescentes y adultos jóvenes hasta los 25 años, encontraron una prevalencia de infección por $C$. trachomatis de 3,7\% en hombres y 4,7\% en mujeres, utilizando reacción en cadena de la ligasa en muestras de orina. ${ }^{16}$ En un estudio poblacional en Holanda, entre adolescentes de 15 a 19 años, se encontró una prevalencia de infección por $C$. trachomatis de $2,5 \%$ en mujeres y $1,5 \%$ en hombres, utilizando la técnica de PCR en muestras de orina. ${ }^{17}$

Gencay y colaboradores encontraron 5 mujeres positivas para Chlamydia entre 14 mujeres con parto prematuro, a quienes les realizaron PCR en muestras de secreción cervical ${ }^{18}$ e Ismail y colaboradores evaluaron 178 pacientes con RPM pretérmino (entre las 22 y las 35 semanas de gestación) encontrando una prevalencia de $C$. trachomatis del 14,6\%. ${ }^{19}$ En el estudio John Hopkins de cervicitis y resultados adversos del embarazo, se encontró que la colonización con $C$. trachomatis se asoció significativamente con retardo de crecimiento intrauterino (OR 2,4; IC95\% 1,32 - 4,18) y parto prematuro (OR 1,6; IC95\% 1,01 - 2,5). ${ }^{20}$ Debido a los desenlaces perinatales adversos señalados se ha sugerido hacer detección y tratamiento de 
las infecciones por Chlamydia trachomatis en las mujeres gestantes. ${ }^{21,22}$

Aunque se dispone de varios métodos para la detección de $C$. trachomatis en especímenes clínicos, la introducción de pruebas de amplificación de DNA, proveen la oportunidad de usar muestras no invasivas y de fácil recolección. El método utilizado en nuestro estudio fue la PCR. ${ }^{9,23}$ La técnica de PCR alcanza una sensibilidad del 98\%, gracias a que el límite inferior de detección es de 1 a 10 cuerpos elementales. La especificidad del PCR es del 97 al 100\%. La técnica Cobas Amplicor, detecta correctamente la presencia de Chlamydia trachomatis en muestras de orina en un 97\%. ${ }^{24}$ Además de la alta sensibilidad y especificidad del PCR, la detección casi simultánea con el evento adverso tiene ventaja sobre los estudios serológicos, ya que éstos no determinan el momento de la infección. No obstante, es posible que se requieran muestras simultáneas de orina y de endocérvix para investigar por PCR, la verdadera prevalencia. ${ }^{25}$

Las demás pruebas para detección de C. trachomatis tienen menor sensibilidad y especificidad que el PCR. Los cultivos tienen una sensibilidad del 70 a 90\% y especificidad 100\%, no están ampliamente disponibles y tienen una alta tasa de falsos negativos cuando se comparan con las pruebas de amplificación de ácidos nucleicos. ${ }^{26,27}$ La detección del microrganismo por inmunofluorescencia (DFA por sus siglas en inglés) tiene una sensibilidad del 70 al 100\% y una especificidad mayor a 95\% y la detección de antígenos por inmunoanálisis (EIA por sus siglas en inglés) tiene una sensibilidad del 43 al $92 \%$ y una especificidad del 92 al 100\%. ${ }^{28}$ Nuestros datos pueden ser extrapolados a gestantes de alto riesgo perinatal dado que se trata de una institución de referencia nacional de obstetricia nivel IV.

En conclusión nuestros datos sugieren que la $C$. trachomatis debe investigarse en pacientes con trabajo de parto prematuro o con RPM pretérmino, aún en ausencia del antecedente de flujo genital patológico. La detección puede ser particularmente importante en mujeres con alto riesgo de parto prematuro (antecedente de parto prematuro y de RPM pretérmino) y en quienes no se encuentra la causa de trabajo de parto prematuro usando los métodos diagnósticos rutinarios en nuestro medio.

\section{REFERENCIAS}

1. Mandell GL, Gordon DR, Bennett JE. Mandell, Douglas, and Bennett's principles and practice of infectious diseases. 6th ed. Philadelphia: Elsevier Churchill Livingstone; 2005.

2. Datta SD, Sternberg, M, Johnson R et al. Prevalence of chlamydia and gonorrhea in the United States among persons aged 14-39 years, 1999-2000. Program and abstracts of the 15 th Annual Meeting of the International Society of Sexually Transmitted Disease Research; 2003 July 27-30; Ottawa, Ontario, Canada. Abstract 349.

3. King J, Flenady V. Antibiotics for preterm labour with intact membranes. Cochrane Database Syst Rev 2000;(2):CD000246. Update in: Cochrane Database Syst Rev 2002;(4).

4. Gaitán H, Eslava J, Garzón CD, Rubio JA, Forero J, Valbuena G, et al. Tamizaje de eventos adversos en atención obstétrica y del puerperio en el Instituto Materno Infantil de Bogotá, Colombia. 2002-2003. Rev Colomb Obstet Ginecol 2005;56:18-27.

5. Rivlin ME, Morrison JC, Grossman JH 3rd. Comparison of pregnancy outcome between treated and untreated women with chlamydial cervicitis. J Miss State Med Assoc 1997;38:404-7.

6. Fejgin MD, Cohen I, Horvat-Kohlmann M, Charles AG, Luzon A, Samra Z. Chlamydia trachomatis infection during pregnancy: can it cause an intrauterine infection? Isr J Med Sci 1997;33:98-102.

7. Ngassa PC, Egbe JA. Maternal genital Chlamydia trachomatis infection and the risk of preterm labor. Int J Gynaecol Obstet 1994;47:241-6.

8. Black CM. Current methods of laboratory diagnosis of Chlamydia trachomatis infections. Clin Microbiol Rev 1997;10:160-84.

9. Johnson RE, Green TA, Schachter J, Jones RB, Hook EW 3rd, Black CM, et al. Evaluation of nucleic acid amplification tests as reference tests for Chlamydia trachomatis infections in asymptomatic men. J Clin Microbiol 2000;38:4382-6. 
10. Cook RL, Hutchison SL, Ostergaard L, Braithwaite RS, Ness RB. Systematic review: noninvasive testing for Chlamydia trachomatis and Neisseria gonorrohoeae. Ann Inter Med 2005;142:914-25.

11. WHO, 2001. Global Prevalence and Incidence of Selected Curable Sexually Transmitted Infections: Overview and Estimates. Geneva: WHO.

12. Fenton KA, Mercer CH, Johnson AM, Byron CL, McManus S, Erens B, et al. Reported sexually transmitted disease clinic attendance and sexually transmitted infections in britain: prevalence, risk factors, and proportionate population burden. J Infect Dis 2005;191 Suppl 1:S127-38.

13. Cravioto MdelC, Matamoros O, Villalobos-Zapata Y, Pena O, Garcia-Lara E, Martinez M, et al. Prevalence of anti-Chlamydia trachomatis and anti-Neisseria gonorrhoeae antibodies in Mexican populations. Salud Publica Mex 2003;45 Supp 5:S681-9.

14. Jones RB, Katz BP, van der Pol B, Caine VA, Batteiger BE, Newhall WJ. Effect of blind passage and multiple sampling on recovery of Chlamydia trachomatis from urogenital specimens. J Clin Microbiol 1986;24:102933.

15. Verkooyen RP, Luijendijk A, Huisman WM, Goessens WH, Kluytmans JA, van Rijsoort-Vos JH, et al. Detection of PCR Inhibitors in cervical specimens by using the AMPLICOR Chlamydia trachomatis assay. J Clin Microbiol 1996;34:3072-4.

16. Miller WC, Ford CA, Morris M, Handcock MS, Schmitz JL, Hobbs MM, et al. Prevalence of chlamydial and gonococcal infections among young adults in the United States. JAMA 2004;291:2229-36.

17. Van Bergen J, Götz HM, Richardus JH, Hoebe CJ, Broer J, Coenen AJ; PILOT CT study group. Prevalence of urogenital Chlamydia trachomatis increases significantly with level of urbanisation and suggests targeted screening approaches: results from the first national population based study in the Netherlands. Sex Transm Infect 2005; 81:17-23.

18. Gencay M, Koskiniemi M, Fellman V, Ammala P, Vaheri A, Puolakkainen M. Chlamydia trachomatis infection in mothers with preterm delivery and in their newborn infants. APMIS 2001;109:636-40.

19. Ismail MA, Pridjian G, Hibbard JU, Harth C, Moawad AA. Significance of positive cervical cul- tures for Chlamydia trachomatis in patients with preterm premature rupture of membranes. Am J Perinatol 1992;9:368-70.

20. The John Hopkins Study of Cervicitis and Adverse Pregnancy Outcome. Association of Chlamydia trachomatis and Mycoplasma hominis with intrauterine growth retardation and preterm delivery. Am J Epidemiol 1989;129:1247-57.

21. Nyari T, Deak J, Nagy E, Vereb I, Kovacs L, Meszaros G, et al. Epidemiological study of Chlamydia trachomatis infection in pregnant women in Hungary. Sex Transm Infect 1998;74:213-5.

22. Rastogi S, Das B, Salhan S, Mittal A. Effect of treatment for Chlamydia trachomatis during pregnancy. Int J Gynaecol Obstet 2003;80:129-37.

23. Matthews-Greer JM, McRae KL, LaHaye EB, Jamison RM. Validation of the Roche COBAS Amplicor system for Chlamydia trachomatis. Clin Lab Sci $2001 ; 14: 82-4$.

24. Verkooyen RP, Noordhoek GT, Klapper PE, Reid J, Schirm J, Cleator GM, et al. Reliability of nucleic acid amplification methods for detection of Chlamydia trachomatis in urine: results of the first international collaborative quality control study among 96 laboratories. J Clin Microbiol 2003;41:3013-6.

25. Martin DH, Nsuami M, Schachter J, Hook EW 3rd, Ferrero D, Quinn TC, et al. Use of multiple nucleic acid amplification tests to define the infected-patient "gold standard" in clinical trials of new diagnostic tests for Chlamydia trachomatis. J Clin Microbiol 2004; 42:4749-58.

26. Mariani SM. Molecular diagnosis of common STDs. Conference Report. Annual Meeting of the American Society of Clinical Pathology. September 18-21, 2003; New Orleans, Louisiana. Medscape Molecular Medicine 2003: 5(2).

27. Jaschek, G, Gaydos CA, Welsh LE, Quinn TC. Direct detection of Chlamydia trachomatis in urine specimens from symptomatic and asymptomatic men by using a rapid polymerase chain reaction assay. J Clin Microbiol 1993;31:1209-12.

28. Harindra V, Underhill G, Tobin JM. Screening for genital chlamydia infection: DNA amplification techniques should be the test of choice. Int J STD AIDS 2003;14:723-6. 